\section{BMJ Open Respiratory Research}

\title{
Relationship of Pneumocystis antibody responses to paediatric asthma severity
}

\author{
Emily Rayens, ${ }^{1}$ Brenda Noble, ${ }^{1}$ Alfin Vicencio, ${ }^{2}$ David L Goldman, ${ }^{3}$ \\ Supinda Bunyavanich, ${ }^{2,4}$ Karen A Norris ${ }^{1}$
}

To cite: Rayens E, Noble B, Vicencio A, et al. Relationship of Pneumocystis antibody responses to paediatric asthma severity. BMJ Open Resp Res 2021;8:e000842. doi:10.1136/ bmjresp-2020-000842

Received 20 November 2020 Revised 9 March 2021 Accepted 10 March 2021
Check for updates

C) Author(s) (or their employer(s)) 2021. Re-use permitted under CC BY-NC. No commercial re-use. See rights and permissions. Published by BMJ.

${ }^{1}$ Center for Vaccines and Immunology, University of Georgia College of Veterinary Medicine, Athens, Georgia, USA

${ }^{2}$ Pediatrics, Division of Pediatric Pulmonology, Icahn School of Medicine at Mount Sinai, New York, New York, USA

${ }^{3}$ Departments of Pediatrics, Microbiology \& Immunology, Children's Hospital at

Montefiore, Bronx, New York, USA

${ }^{4}$ Department of Genetics and Genomic Sciences, Icahn School of Medicine at Mount Sinai, New York, New York, USA

Correspondence to Dr Karen A Norris; kanorris@uga.edu

\section{ABSTRACT}

Background Although asthma is the most commonly diagnosed respiratory disease, its pathogenesis is complex, involving both genetic and environmental factors. A role for the respiratory microbiome in modifying asthma severity has been recently recognised. Airway colonisation by Pneumocystis jirovecii has previously been associated with multiple chronic lung diseases, including chronic obstructive pulmonary disease (COPD) and severe asthma (SA). Decreased incidence of Pneumocystis pneumonia in HIV-infected individuals and reduced severity of COPD is associated with naturally occurring antibody responses to the Pneumocystis antigen, Kexin (KEX1).

Methods 104 paediatric patients were screened for KEX1 IgG reciprocal end point titre (RET), including 51 with $S A$, 20 with mild/moderate asthma, 20 non-asthma and 13 with cystic fibrosis (CF) in a cross-sectional study. Results Patients with SA had significantly reduced Pneumocystis KEX1 titres compared with patients with mild/moderate asthma $(p=0.018)$ and $C F(p=0.003)$. A binary KEX1 RET indicator was determined at a threshold of KEX1 RET $=1000$. Patients with SA had $4.40(95 \% \mathrm{Cl}$ 1.28 to $13.25, \mathrm{p}=0.014)$ and $17.92(95 \% \mathrm{Cl} 4.15$ to 66.62 , $\mathrm{p}<0.001$ ) times the odds of falling below that threshold compared with mild/moderate asthma and patients with CF, respectively. Moreover, KEX1 IgG RET did not correlate with tetanus toxoid $\lg G(r=0.21, p=0.82)$ or total $\lg E(r=0.03, p=0.76)$, indicating findings are specific to antibody responses to KEX1.

Conclusions Paediatric patients with SA may be at higher risk for chronic Pneumocystis infections and asthma symptom exacerbation due to reduced levels of protective antibodies. Plasma KEX1 lgG titre may be a useful parameter in determining the clinical course of treatment for paediatric patients with asthma.

\section{INTRODUCTION}

Asthma is the most commonly diagnosed chronic respiratory disease worldwide, affecting over 300 million people of all ethnic groups and ages. ${ }^{1}$ Patients diagnosed with the asthma subphenotype severe asthma (SA) exhibit sustained symptoms including breathlessness, wheezing and coughing despite high levels of inhaled or oral corticosteroids and bronchodilators. ${ }^{2}$ While some genetic ${ }^{3}$ and environmental factors ${ }^{4}$ have been shown to contribute to the development of asthma, the
Key messages

Are antibody titres to the Pneumocystis (Pc) protein Kexin (KEX1), which have been previously correlated with a failure to control Pc infection in patients with HIV or chronic obstructive pulmonary disease (COPD), reduced in patients with severe asthma (SA) compared with those with mild or moderate disease?

- Paediatric patients with SA had significantly reduced antibody titres to KEX1 compared with patients with mild/moderate asthma or cystic fibrosis, suggesting that these patients may have chronic underlying Pc infections contributing to their severe symptoms and, further, that low anti-KEX1 titre is a marker for SA.

- This is the first study to demonstrate that patients with SA have reduced levels of the protective Pc antibody KEX1, generating new diagnostic and therapeutic possibilities centred on the potential role of underlying $\mathrm{Pc}$ infection in exacerbation of $\mathrm{SA}$ symptoms.

role of infectious agents in development and exacerbation of chronic disease continues to evolve. Fungal pathogens have only recently being started appreciated for their potential role in asthma symptoms, despite infectioninduced production of mucin and $\mathrm{T}_{\mathrm{H}} 2$ related cytokines directly promoting asthmatic processes. ${ }^{5}$ Animal models have demonstrated that colonisation with fungal pathogens, such as Aspergillus fumigatus and, more recently, Pneumocystis jirovecii (Pc), contribute to asthma-like phenotypes, including airway hyper-responsiveness. ${ }^{6}$

$\mathrm{Pc}$ is an opportunistic fungal pathogen and a causative agent of pneumonia in immunocompromised individuals. Pc has also been previously implicated in chronic lung disease, including chronic obstructive pulmonary disease (COPD); Pc colonisation was associated with the development and severity of COPD in human subjects and animal models. The role of Pc in chronic lung disease has been further expanded with recent findings where Pc was found to be enriched in the lung 
microbiota of patients with SA and COPD compared with patients without asthma and COPD, respectively. ${ }^{89}$

While host defence against Pc involves both humoral and cellular immune responses, our group has shown that antibody responses to an internal 90 amino acid peptide of the Pneumocystis endoprotease Kexin (KEX1) correlate with protection from Pc pneumonia in HIVinfected individuals and that protection can be induced through vaccination with KEX1 recombinant protein in a non-human primate model of HIV infection. ${ }^{1011}$ Furthermore, low or undetectable KEX1 antibody titres were an independent predictor of disease severity in patients with COPD ${ }^{12}$ suggesting a role for anti-KEX1 antibodies in preventing the deleterious effects of Pc colonisation.

Pc colonisation induces chronic inflammation and has been implicated as a co-factor in chronic lung diseases such as COPD and SA, and anti-KEX1 antibodies are associated with protection against Pc infection. Thus, we sought to determine whether paediatric patients with SA had reduced levels of protective Pc antibodies compared with paediatric patients with other respiratory diagnoses, including mild/moderate asthma. We performed a cross-sectional pilot study of anti-KEX1 antibody titres in cohorts of patients with SA, mild/moderate asthma, nonasthma (NA) and cystic fibrosis (CF).

\section{METHODS}

\section{Cohort description}

Plasma collected from paediatric patients with SA $(n=51)$ was analysed against that collected from cohorts of children with mild/moderate asthma $(n=20)$ and $C F(n=13)$, as well as a NA cohort $(n=20)$. In additional to the NA cohort, patients with CF were used as a control for SA as both are chronic pulmonary conditions associated with small airway obstruction.

Plasma samples were collected from patients receiving care at Cohen's Children's hospital and the Mount Sinai Kravis Children's Hospital. The diagnosis of asthma was confirmed with either methacholine challenge or prealbuterol and post-albuterol spirometry to document airway reactivity/reversibility. Severe asthma was defined by SARP criteria, ${ }^{13}$ while mild/moderate was defined based on $\geq 2$ symptoms per week, use of an inhaled bronchodilator $\geq$ twice weekly or use of daily medication for asthma and increased airway responsiveness to methacholine (PC20 $\leq 12.5 \mathrm{mg} / \mathrm{mL}) .{ }^{14}$ All control patients had no personal or family history of asthma in first-degree relatives, normal spirometry and no bronchodilator response.

IgE levels to specific allergens, including Aspergillus, were determined from a commercial lab as part of routine care.

\section{KEX1 reciprocal end point antibody titre determination}

A 90 amino acid fragment of human-derived KEX1 was cloned into the pET28b (+) expression vector (GenScript) in Escherichia coli BL21(DE3) pLysS (Thermo Fisher
Scientific) and used to produce an approximately 11 $\mathrm{kDa}$ recombinant protein. Microtitre plates (Immunolon 4HBX; Thermo Fisher Scientific) were coated with $5 \mu \mathrm{g} /$ $\mathrm{mL}$ of purified KEX1 in phosphate-buffered saline (PBS). Heat-inactivated plasma was diluted 1:100 in blocking buffer (PBS with $5 \%$ non-fat milk); $50 \mu \mathrm{L}$ of plasma were plated into KEX1-coated wells, and serial dilutions were made to determine end point titres. Goat antihuman immunoglobulin-conjugated horseradish peroxidase (1:10 000, IgG; Sigma-Aldrich) was used for detection, and plates were developed by standard methods. Plasma samples from this cohort that had an undetectable Pc antibody titre were used as negative controls. The reciprocal end point titre (RET) was calculated as the highest dilution at which the optical density was the same or less than that of the negative control. To determine whether patients with low anti-KEX1 antibody titres had a generalised defect in humoral immunity, tetanus toxoid antibody levels were determined using commercial ELISA kit (MyBioSource).

\section{Statistical analysis}

All statistical analyses were performed using GraphPad Prism (GraphPad Software, La Jolla, California, USA). An alpha of 0.05 was used for inferential testing. Cohort demographic and clinical characteristics were compared among the four cohorts using one-way analysis of variance (ANOVA) or Fisher's exact test, as appropriate. Samples without a detectible KEX1 titre were assigned a value of 50 , which is half the limit of detection. Independent sample t-tests were used to compare the KEX1 and tetanus toxoid results of the severe asthma cohort to each of the others with a Holm correction for multiple comparisons. ${ }^{15}$ For a set of three comparisons, this correction compares the smallest observed $p$ value to 0.017 , the middle observed $p$ value to 0.025 and the largest to 0.05 . ANOVA was not used for the KEX1 comparisons since the emphasis was on SA versus each of the other cohorts, rather than an assessment of differences among all four groups. The Baptista-Pike method was used to calculate ORs and their corresponding 95\% CIs. Fisher's exact test was used to determine association between pulmonary diagnosis and low KEX1 RET.

\section{Patient and public involvement}

Patients were not involved in the design, conduct or interpretation of this post hoc analysis.

\section{RESULTS}

One hundred four paediatric patients were included in the analysis: 51 diagnosed with severe asthma, 20 diagnosed with mild/moderate asthma, 13 diagnosed with CF and 20 categorised as NA. Patient cohorts were similar with regard to age $(\mathrm{p}=0.407)$ and sex $(\mathrm{p}=0.895)$, as seen in table 1. Total IgE titre, collected as part of routine care, was also compared across cohorts and found to be 


\begin{tabular}{|c|c|c|c|c|}
\hline Cohort & Severe asthma & $\begin{array}{l}\text { Mild and moderate } \\
\text { asthma }\end{array}$ & Non-asthma & Cystic fibrosis \\
\hline All patients & $n=51$ & $n=20$ & $n=20$ & $n=13$ \\
\hline Male, sex, N (\%) & 29 (56.9) & $11(55.0)$ & $12(60.0)$ & $7(53.8)$ \\
\hline \multicolumn{5}{|l|}{ Race, N (\%) } \\
\hline White & $23(45.1)$ & $10(50.0)$ & $18(90.0)$ & $13(100.0)$ \\
\hline Black & $12(23.5)$ & $5(25.0)$ & $0(0.0)$ & $0(0.0)$ \\
\hline Asian & $2(3.9)$ & $1(5.0)$ & $0(0.0)$ & $0(0.0)$ \\
\hline More than one & $11(21.6)$ & $4(20.0)$ & $1(5.0)$ & $0(0.0)$ \\
\hline Unknown & $3(5.9)$ & $0(0.0)$ & $1(5.0)$ & $0(0.0)$ \\
\hline Ethnicity: Hispanic/Latinx & $9(17.6)$ & $6(30.0)$ & $1(5.0)$ & $2(15.4)$ \\
\hline Age, years $($ mean $\pm S D)$ & $10.9 \pm 4.2$ & $11.9 \pm 3.6$ & $11.9 \pm 3.6$ & $12.9 \pm 3.6$ \\
\hline Total lgE titre (log) & $2.2 \pm 1.0$ & $2.4 \pm 0.4$ & $2.6 \pm 0.4$ & $1.7 \pm 0.8$ \\
\hline Used inhaled corticosteroids, N (\%) & $51(100.0)$ & $0(0.0)$ & $0(0.0)$ & $0(0.0)$ \\
\hline
\end{tabular}

similar between the groups $(p=0.529)$. Cohorts varied by racial breakdown, but were predominantly white. No significant differences in KEX1 titres were found by racial group ( $\mathrm{p}=0.844$ ). All patients with SA were on inhaled corticosteroid regimens, while the patients with mild/ moderate asthma, NA and CF were not.

Figure 1A shows that patients diagnosed with SA had significantly reduced KEX1 IgG antibody RETs compared with patients with mild/moderate asthma $(\mathrm{p}=0.018)$ and CF ( $p=0.003$ ), but did not differ significantly for patients with NA $(p=0.077)$. Similar analyses for tetanus toxoid antibody titres in patients with SA demonstrated no significant relationship when compared with patients with mild/moderate asthma $(\mathrm{p}=0.522), \mathrm{CF}(\mathrm{p}=0.171)$ or NA (0.253), suggesting the lower KEX1 RET in the SA cohort was not a generalised defect in the humoral response (figure 1B).

The mean KEX1 antibody titre across cohorts was 953 (range 1-32 000) and thus a cut-off of 1000 was specified for a low KEX1 antibody titre. Within the respiratory disease status groups, $16 \%$ of patients with SA (8 of 51 ), $5 \%$ of patients with mild/moderate ( 1 of 20 ), $20 \%$ of patients with NA (4 of 20) and $0 \%$ of patients with CF (0 of 13) had undetectable titres. When characterised by high and low KEX1 antibody titres, $84.3 \%$ (43 of 51 ) of patients with SA had a low KEX1 titre while only $55 \%$ (11 of 20) of patients with mild/moderate asthma, $60 \%$ of patients with NA (12 of 20) and $23.1 \%$ (3 of 13) of patients with CF had low KEX1. Using the binary KEX1 RET indicator, table 2 shows patients with SA had 4.40 times the odds of a low KEX1 RET compared with patients with mild/moderate asthma (95\% CI 1.23 to $13.25, \mathrm{p}<0.05)$ and 17.92 times the odds compared with patients with CF (95\% CI 4.15 to $66.62, \mathrm{p}<0.0001)$. The odds of a low KEX1 RET among participants with SA were 3.58 compared with the NA cohort; however, this association was not quite significant (95\% CI 1.20 to $10.84, \mathrm{p}=0.055$ ).

Figure 2 demonstrates the specificity these findings in anti-KEX1 antibodies as they relate to pulmonary disease diagnosis. Figure 2A shows that there is no significant correlation between KEX1 IgG RET and tetanus toxoid $\operatorname{IgG}(\mathrm{r}=0.21, \mathrm{p}=0.82)$, supporting the findings that low KEX1 is not the result of a generalised humoral defect across cohorts. KEX1 IgG RET was also not significantly correlated with total $\operatorname{IgE}$ titre $(\mathrm{r}=0.03, \mathrm{p}=0.76)$, indicating that the titres observed were not part of a broader, nonspecific antibody response.
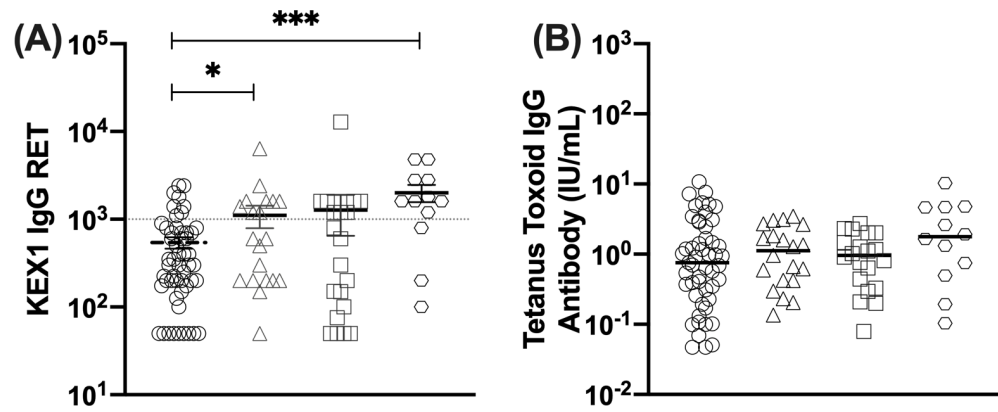

- Severe Asthma $(n=51)$

$\triangle$ Mild/Moderate $(n=20)$

Non-Asthma $(\mathrm{n}=20)$

- Cystic Fibrosis $(n=13)$

Figure 1 Plasma immunoglobulin titres. (A) Kexin (KEX1)-specific IgG titre as determined by ELISA and (B) tetanus toxoid antibody (IU/mL), stratified by disease diagnosis. Low KEX1 titre cut-off indicated by dotted line. ${ }^{*} \mathrm{P}<0.05,{ }^{* \star *} \mathrm{p}<0.001$. RET, reciprocal end point titre. 


\begin{tabular}{|c|c|c|c|c|}
\hline Cohort & Severe asthma & Mild and moderate asthma & Non-asthma & Cystic fibrosis \\
\hline All patients & $\mathrm{n}=51$ & $n=20$ & $n=20$ & $n=13$ \\
\hline \multicolumn{5}{|l|}{ Primary outcome } \\
\hline High KEX1 RET & 8 & 9 & 8 & 10 \\
\hline Low KEX1 RET & 43 & 11 & 12 & 3 \\
\hline \multicolumn{5}{|c|}{ OR of low KEX1 RET in patients with severe asthma } \\
\hline Value & - & 4.40 & 3.58 & 17.92 \\
\hline 95\% Cl, Baptista-Pike & - & 1.28 to 13.25 & 1.20 to 10.84 & 4.15 to 66.62 \\
\hline \multicolumn{5}{|c|}{ Association between pulmonary diagnosis and binary KEX1 RET indicator } \\
\hline Fisher's exact test $p$ value & - & $0.014^{*}$ & 0.055 & $<0.0001^{\star \star *}$ \\
\hline
\end{tabular}

${ }^{*} \mathrm{P}<0.05,{ }^{* \star *} \mathrm{p}<0.001$.

KEX1, Kexin; OR, Odds Ratio; RET, reciprocal end point titre.

\section{DISCUSSION}

This study is the first to report on the relationship of Pc KEX1 titres and paediatric asthma diagnosis. We found that patients with SA have significantly reduced KEX1 IgG titres compared with patients with mild/moderate asthma and CF. When a binary KEX1 RET indicator was used to categorise titres as 'high' and 'low', patients with SA had 4.4 and 17.9 times the odds of having a low KEX1 RET compared with patients with mild/moderate asthma and $\mathrm{CF}$, respectively.

Comparable tetanus toxoid antibody levels in these cohorts suggest that the lower KEX1 antibody titres in patients with SA is not an indicator of generally poorer humoral immunity within the cohort. This conclusion is further supported by the correlation analysis of KEX1 titres with tetanus toxoid and total IgE titres. The lack of correlation between KEX1 RET and tetanus toxoid antibody titres further indicates that differences in KEX1 RET are not a result of a generalised humoral defect across or within groups. Furthermore, elevated IgE titres do not correlate with elevated KEX1 RET, indicating that differences in KEX1 titres are distinct from overall antibody responses associated with allergens (including fungi). ${ }^{16}$ These findings support the hypothesis that reduced Pc KEX1 antibody titres indicate a reduced capacity to control Pc infection and may lead to symptom exacerbation in patients with asthma.

Previous studies have shown a relationship between Pc colonisation and chronic lung diseases, including SA. ${ }^{6}{ }^{12}$ Patients with SA have increased enrichment of Pc in bronchoalveolar lavage fluid (BALF $)^{8}$ and higher sera titres against whole cell Pc antigens, ${ }^{6}$ suggesting increased exposure to Pc in patients with SA compared with healthy individuals. However, there are multiple Pc antigens which are highly immunogenic but not associated with protection from infection, ${ }^{10}$ and thus we sought

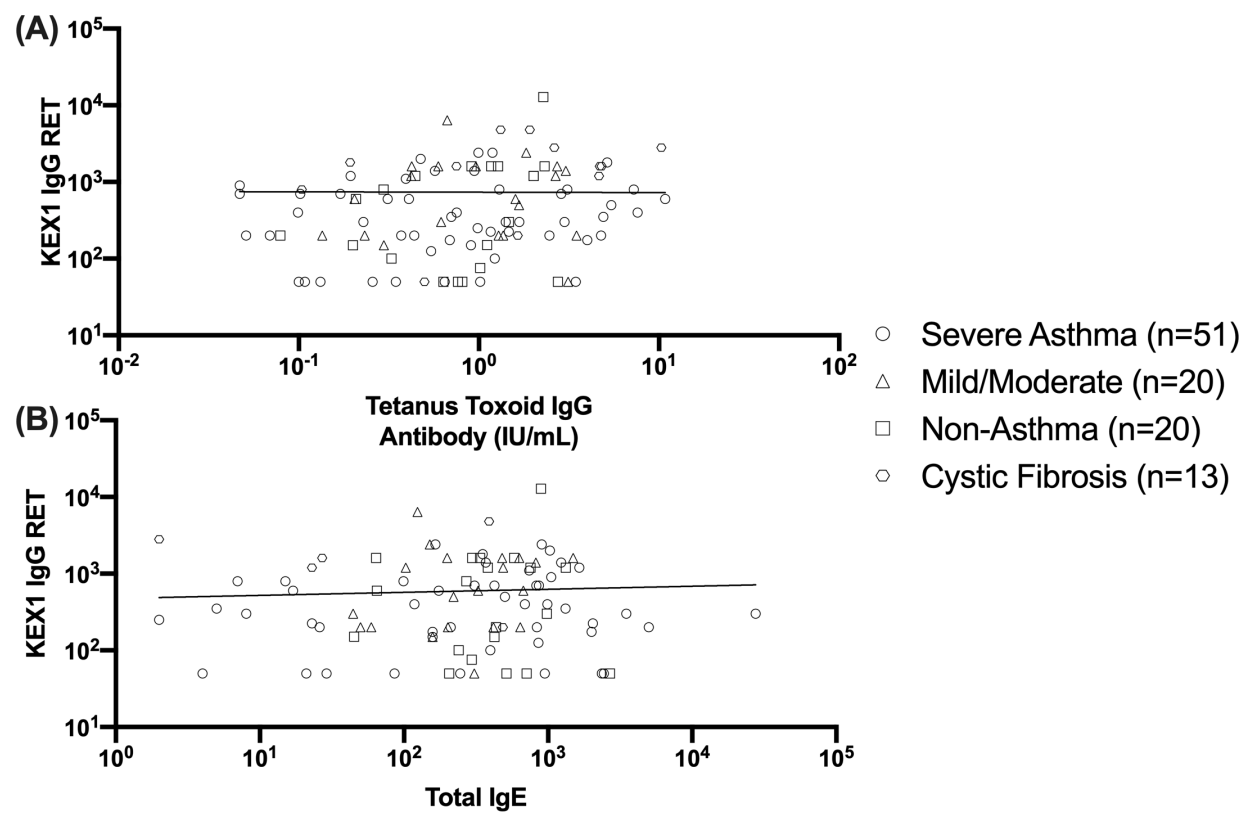

Figure 2 Correlation of antibody responses. (A) Correlation between Kexin (KEX1) IgG reciprocal end point titre (RET) and tetanus toxoid $\lg G(r=0.21, p=0.82)$ and $(B)$ correlation between $K E X 1 \lg G$ RET and total $\lg E(r=0.03, p=0.76)$. 
to determine the relationship between SA and immunity to the protective Pc antigen KEX1. ${ }^{17}$ The association of low anti-KEX1 antibody levels expands the findings of previous studies, which implicate anti-KEX1 antibody responses in protection against Pc pneumonia and COPD. ${ }^{7}$ In human and non-human primate models of HIV, low anti-KEX1 titres was predictive of susceptibility to PCP. ${ }^{10}{ }^{11}$ Additionally, in an examination of patients with COPD, undetectable anti-KEX1 antibody titres were an independent predictor of more severe airway obstruction in smokers. ${ }^{12}$

This study had several limitations. Antibody levels were measured at a single time and we did not have corresponding data on colonisation with Pc in these cohorts. Thus, we were unable to determine the cause and effect of Pc colonisation with SA. As we have shown in COPD, persistence of this organism in the lung environment may amplify the inflammatory response and worsen disease or be an indicator of disease severity. ${ }^{12} 18$ Additionally, the cohorts differed in demographic factors, although those differences were not significant. A larger study would allow a more in-depth consideration of demographic covariates. These groups did, however, significantly differ in corticosteroid use. All patients with SA were on inhaled corticosteroid regimens while the patients with mild/moderate asthma, CF and NA were not, although this difference did not appear to affect tetanus toxoid or IgE antibody levels and suggests that this likely does not play a role in KEX1 differences. Despite these limitations, these results provide the basis for further investigation of the role of Pc colonisation in SA and the potential protective effect of anti-KEX1 humoral responses is preventing or ameliorating SA symptoms. Further studies linking Pc colonisation, pulmonary inflammatory responses and the potential use of anti-KEX1 antibody responses as a biomarker of disease progression will be informative.

These findings have a number of potential clinical implications. Despite the heterogeneity of paediatric asthma (ie, viral-induced, allergic, non-allergic), as well as its diversity in underlying genetic and environmental factors, current treatment is limited to anti-inflammatory and bronchodilator therapies. Even for patients who fail maximum doses of inhaled corticosteroids and longacting beta agonists, alternate approaches which also target the inflammatory response (specifically anti-IL5 or anti-IgE monoclonal antibodies) represent the only FDA-approved options. Although shown to be effective in controlling symptoms for patients with severe-persistent disease, such therapies lack long-term safety data and have unfavourable financial implications. Importantly, our findings highlight the possibility of targeted antimicrobial therapy as a potentially feasible and inexpensive option to improve symptom control, and perhaps lower requirements for standard therapies. Furthermore, because children may in fact be a significant reservoir for $\mathrm{Pc},{ }^{19}$ these findings may be important to consider for family members or close contacts with asthma or other conditions who have a high risk for Pc infection.
In summary, we found that patients with SA have a reduced anti-KEX1 Pc titre compared with patients with mild/moderate asthma and CF. These findings lend additional support to a potential role for Pc colonisation in the pathogenesis of severe asthma and suggest that protective anti-KEX1 antibody responses might be an important mechanism by which Pc colonisation is reduced or prevented. Furthermore, linking KEX1 antibody response to detection of Pc colonisation in BALF and asthma disease severity will be informative in determining whether identification and antibiotic treatment of Pc colonisation represents a therapeutic option for paediatric patients with SA. Future studies may also validate the use of plasma anti-KEX1 titres as a non-invasive marker for the identification of patients susceptible to chronic Pc colonisation and asthma exacerbation.

Contributors All authors of this manuscript were involved in the acquisition, analysis or interpretation of the data, and in the preparation or critical revision of the manuscript. All authors approved the final version of the manuscript.

Funding This work was supported by Georgia Research Alliance (KAN), the University of Georgia Research Foundation (KAN) and the National Institutes of Health grant number Al118833 (SB).

Competing interests None declared.

Patient and public involvement Patients and/or the public were not involved in the design, or conduct, or reporting, or dissemination plans of this research.

Patient consent for publication Not required.

Ethics approval Patient samples were banked under Institutional Review Boardapproved studies at Cohen's Children Hospital in Queens, New York, USA and the Mount Sinai Kravis Children's Hospital, New York, New York, USA. Participants provided informed consent (including consent for future studies).

Provenance and peer review Not commissioned; externally peer reviewed.

Data availability statement Data are available on reasonable request. All data relevant to the study are included in the article.

Open access This is an open access article distributed in accordance with the Creative Commons Attribution Non Commercial (CC BY-NC 4.0) license, which permits others to distribute, remix, adapt, build upon this work non-commercially, and license their derivative works on different terms, provided the original work is properly cited, appropriate credit is given, any changes made indicated, and the use is non-commercial. See: http://creativecommons.org/licenses/by-nc/4.0/.

\section{REFERENCES}

1 SorianoJB, AbajobirAA, AbateKH, et al. Global, regional, and national deaths, prevalence, disability-adjusted life years, and years lived with disability for chronic obstructive pulmonary disease and asthma, 1990-2015: a systematic analysis for the global burden of disease study 2015. Lancet Respir Med 2017;5:691-706.

2 Guilbert TW, Bacharier LB, Fitzpatrick AM. Severe asthma in children. J Allergy Clin Immunol Pract 2014;2:489-500.

3 Moffatt MF, Gut IG, Demenais F, et al. A large-scale, consortiumbased genomewide association study of asthma. N Engl J Med 2010;363:1211-21.

4 Hammad H, Lambrecht BN. Barrier epithelial cells and the control of type 2 immunity. Immunity 2015;43:29-40.

5 Goldman DL, Huffnagle GB. Potential contribution of fungal infection and colonization to the development of allergy. Med Mycol 2009;47:445-56.

6 Eddens T, Campfield BT, Serody K, et al. A Novel CD4 ${ }^{+}$T CellDependent Murine Model of Pneumocystis-driven Asthma-like Pathology. Am J Respir Crit Care Med 2016;194:807-20.

7 Morris A, Sciurba FC, Norris KA. Pneumocystis: a novel pathogen in chronic obstructive pulmonary disease? COPD 2008;5:43-51.

8 Goldman DL, Chen Z, Shankar V, et al. Lower airway microbiota and mycobiota in children with severe asthma. J Allergy Clin Immunol 2018;141:e807:808-11. 
9 Cui L, Lucht L, Tipton L, et al. Topographic diversity of the respiratory tract mycobiome and alteration in HIV and lung disease. Am J Respir Crit Care Med 2015;191:932-42.

10 Gingo MR, Lucht L, Daly KR, et al. Serologic responses to Pneumocystis proteins in HIV patients with and without Pneumocystis jirovecii pneumonia. J Acquir Immune Defic Syndr 2011;57:190-6.

11 Kling HM, Norris KA. Vaccine-Induced immunogenicity and protection against Pneumocystis pneumonia in a nonhuman primate model of HIV and Pneumocystis coinfection. J Infect Dis 2016;213:1586-95.

12 Morris A, Netravali M, Kling HM, et al. Relationship of Pneumocystis antibody response to severity of chronic obstructive pulmonary disease. Clin Infect Dis 2008;47:e64-8.

13 Moore WC, Bleecker ER, Curran-Everett D, et al. Characterization of the severe asthma phenotype by the National heart, lung, and blood Institute's severe asthma research program. J Allergy Clin Immunol 2007;119:405-13.
14 Do AN, Chun Y, Grishina G, et al. Network study of nasal transcriptome profiles reveals master regulator genes of asthma. $J$ Allergy Clin Immunol 2021;147:879-93.

15 Aickin M, Gensler H. Adjusting for multiple testing when reporting research results: the Bonferroni vs Holm methods. Am J Public Health 1996;86:726-8.

16 Zhang Z, Reponen T, Hershey GKK. Fungal exposure and asthma: IgE and Non-IgE-Mediated mechanisms. Curr Allergy Asthma Rep 2016;16:86.

17 Ruan S, Cai Y, Ramsay AJ, et al. B cell and antibody responses in mice induced by a putative cell surface peptidase of Pneumocystis murina protect against experimental infection. Vaccine 2017;35:672-9.

18 Shipley TW, Kling HM, Morris A, et al. Persistent Pneumocystis colonization leads to the development of chronic obstructive pulmonary disease in a nonhuman primate model of AIDS. J Infect Dis 2010;202:302-12.

19 Gigliotti F, Wright TW. Pneumocystis: where does it live? PLoS Pathog 2012;8:e1003025. 